\title{
Concomitant central venous sinus thrombosis and subdural hematoma in acute promyelocytic leukemia: middle meningeal artery embolization enables safe anticoagulation. Illustrative case
}

\author{
Kushagra Maini, MD, ${ }^{1}$ Feroze Afzal, MD, ${ }^{1}$ Dan-Victor Giurgiutiu, MD, ${ }^{1}$ Scott Y. Rahimi, MD, ${ }^{2}$ Manan Shah, MD, ${ }^{1}$ Jeffrey A. Switzer, DO, \\ Fernando L. Vale, MD, ${ }^{2}$ and Klepper Alfredo Garcia, MD
}

Departments of ${ }^{1}$ Neurology and ${ }^{2}$ Neurosurgery, Augusta University, Augusta, Georgia

BACKGROUND Acute promyelocytic leukemia (APL) has long been associated with coagulation disorders. The proposed mechanism is a combination of fibrinolysis, proteolysis, platelet dysfunction, thrombocytopenia, and possibly disseminated intravascular coagulation. Hemorrhagic complications are prominent.

OBSERVATIONS In this case, a 25-year-old female with newly diagnosed APL developed extensive cerebral venous thrombosis (CVT) and was initiated on a protocol with idarubicin and all-trans retinoic acid. The general recommendation for treating CVT is anticoagulation to stabilize the existing thrombus and prevent propagation. The patient was initiated on a heparin drip, but her clinical course was complicated by subdural hemorrhage (SDH) and epidural hemorrhage in the setting of thrombocytopenia. Anticoagulation was held, and her CVT propagated on follow-up imaging. To restart anticoagulation for CVT with a limited risk of SDH, the authors pursued middle meningeal artery (MMA) embolization. The patient was transitioned to apixaban and discharged to home.

LESSONS MMA embolization enables safe anticoagulation in patients with concomitant CVT and SDH. The authors report the complex clinical course and effective management of this rare clinical scenario.

https://thejns.org/doi/abs/10.3171/CASE2080

KEYWORDS leukemia; brain bleed; thrombosis; embolization; surgical technique; oncology

Cerebral venous sinus thrombosis is a rare complication of acute promyelocytic leukemia (APL). Some of the common presenting signs of cerebral venous thrombosis (CVT) include headaches, altered mental status, seizure, emesis, visual impairment, and sometimes focal neurological deficits. Coagulopathy in APL is multifactorial, as most patients present with thrombocytopenia, increased levels of fibrin degradation products, and prolongation of thrombin time. ${ }^{1}$ Thrombocytopenia is usually due to bone marrow infiltration by the leukemic cells, further worsened by the myelosuppressive effects of chemotherapy. ${ }^{2}$ All-trans retinoic acid (ATRA), a differentiating agent, is part of the treatment protocol for APL and has been associated with an increased survival rate compared to that with chemotherapy alone. ${ }^{3-6}$ However, ATRA is known to complicate the hematological picture by promoting differential regulation on acute myeloid leukemia $(A M L)$ adhesion molecules, facilitating cell migration and adhesion. ${ }^{7}$ It also enhances the procoagulant properties of endothelium, increasing fatal cerebral thrombosis and myocardial infarctions. ${ }^{5,8,9}$ In a study performed by Escudier et al., ${ }^{10} 3$ of 31 patients were noted to have thrombotic complications during the induction period with ATRA. Nevertheless, ATRA has been shown to be beneficial with a high remission rate and low induction mortality in patients with APL. ${ }^{9,11,12}$ The usual recommendations for CVT are full-dose anticoagulation with unfractionated heparin versus low-molecular-weight heparin, which should be individualized as per the patient's clinical condition and platelet counts. ${ }^{13-15}$ Anticoagulation has been proved to be safe in patients with venous sinus thrombosis, even in the presence of intracerebral hemorrhage in small placebo-controlled studies. ${ }^{16-18}$

\footnotetext{
ABBREVIATIONS AML = acute myeloid leukemia; $A P L=$ acute promyelocytic leukemia; $A T R A=$ all-trans retinoic acid; CT = computed tomography; CVT = cerebral venous thrombosis; $\mathrm{EDH}=$ epidural hemorrhage; $\mathrm{MMA}=$ middle meningeal artery; $\mathrm{MRA}=$ magnetic resonance angiography; $\mathrm{MRI}=$ magnetic resonance imaging; MRV = magnetic resonance venography; SDH = subdural hemorrhage.

INCLUDE WHEN CITING Published June 7, 2021; DOI: 10.3171/CASE2080.
}

SUBMITTED October 23, 2020. ACCEPTED February 14, 2021.

(C) 2021 The authors, CC BY-NC-ND 4.0 (http://creativecommons.org/licenses/by-nc-nd/4.0/). 
To investigate previously published case reports of central venous sinus thrombosis associated with APL, we searched PubMed using the keywords "venous sinus thrombosis" and "APL." This search yielded 9 results. We then searched PubMed using the keywords "venous sinus thrombosis" and "ATRA." This search yielded 5 results. Articles regarding acute myeloblastic leukemia or AML were excluded on the basis of title alone. Articles in languages other than English were excluded as well. Articles potentially meeting criteria for inclusion based on title and abstract were reviewed and included, yielding a total of 7 articles.

\section{Illustrative Case}

\section{History and Examination}

We present a case of a 25-year-old female without a significant medical history who was transferred from an outside hospital for initial laboratory test results raising suspicion of leukemia. She presented with worsening headaches associated with fever/ chills, fatigue, nausea, vomiting, and decreased appetite. Her initial workup included computed tomography (CT) of the head, which was reported as normal; routine laboratory tests; and administration of ondansetron and acetaminophen. Her complete blood count revealed leukocytosis $\left(56,000 / \mathrm{mm}^{3}\right.$ white blood cells with $40,000 / \mathrm{mm}^{3}$ blast cells), prompting urgent transfer to our facility. On admission, the patient underwent workup for acute leukemia and was ultimately diagnosed with APL. She was started on ATRA and arsenic, as well as antimicrobial prophylaxis and dexamethasone. Following the treatment, the patient had a worsening headache along with vertigo and diplopia and was found to have bilateral conjunctival hemorrhages. CT of the head was performed and revealed diffuse cerebral and cerebellar edema without hydrocephalus or herniation. On neurological evaluation, the patient did not have any focal deficits except mild sixth cranial nerve weakness on the right. Follow-up magnetic resonance imaging/magnetic resonance venography (MRI/MRV) of the brain demonstrated nonocclusive thrombus in bilateral transverse sinuses and superior sagittal sinus. The patient was started on a heparin drip without a bolus. One week later, the patient developed sudden-onset nausea and vomiting. CT of the head was repeated and revealed a small epidural hemorrhage (EDH) in the right frontoparietal region and subdural hemorrhage (SDH) of the left convexity, along with a $10-\mathrm{mm}$ left to right midline shift. Heparin drip was discontinued, and the patient was then transferred to the neurology intensive care unit. Heparin-induced thrombocytopenia antibody was negative. Over the next few days, the patient's platelet count continued to drop $\left(10,000 / \mathrm{mm}^{3}\right)$, and she received platelet transfusions. Hematology/oncology recommended discontinuing arsenic and ATRA therapy due to significant thrombocytopenia. The findings of repeat MRV were concerning for clot propagation involving the right transverse sinus. This led to a multidisciplinary approach involving the neurology critical care, neurology, neurosurgery, interventional neurology, and hematology/oncology team, and the decision was made to withhold any further platelet transfusions and perform middle meningeal artery (MMA) embolization to limit the risk of SDH expansion.

\section{Oncological History}

Pathology Results

Fluorescence in situ hybridization results were positive for $\mathrm{t}(15 ; 17)$; flow cytometry findings were positive for aberrant blasts; and the results of a peripheral smear were positive for myeloblasts with figure of 8-shaped inclusions, without granules and rare Auer rods. A diagnosis of APL, hypogranular variant, with high-risk disease was made.

\section{Oncological Treatment}

Hydroxyurea was administered on the 8th of the month, ATRA was administered from the 8th to the 23rd, idarubicin was administered from the 9th to the 11th, and arsenic was administered from the 17 th to the 25 th.

\section{Imaging Findings and Progression}

CT of the head on admission revealed no evidence of an acute intracranial process. CT of the head was again performed 2 days later, when the patient reported worsening of her symptoms. This was concerning for the development of diffuse cerebral and cerebellar edema with associated mass effect without herniation or hydrocephalus.

Follow-up MRI/magnetic resonance angiography (MRA) revealed nonocclusive venous thromboses involving the right transverse sinus and the short segment of the left transverse sigmoid junction along with nonocclusive clots within the superior sagittal sinus.

Ten days later, another CT of the head was performed and revealed mixed SDH and EDH of the left convexity along with a small $\mathrm{EDH}$ in the right frontoparietal region, with a $10-\mathrm{mm}$ left to right midline shift. Follow-up MRA of the head revealed the absence of flow within the distal superior sagittal sinus extending to the sinus confluence, concerning for clot propagation.

Repeat MRA 2 days later revealed no evidence of clot propagation. Soon after, angiography was performed with successful embolization of the left MMA.

CT of the head postembolization revealed stable subacute left subdural hematoma. CT of the head prior to discharge showed stable subacute left lateral temporal subdural hematoma with a 5-mm left to right midline shift. CT of the head at the first follow-up appointment disclosed no evidence of any residual subdural hematoma.

\section{Procedure}

Before the procedure, the technical aspects of the procedure, as well as potential risks and benefits, were explained to the patient. After informed consent was obtained, the patient was brought to the angiography suite and prepped and draped in the usual fashion. The right radial artery was accessed using ultrasound guidance, followed by the infusion of nitroglycerin $200 \mu \mathrm{g}$ and verapamil $2.5 \mathrm{mg}$ mixed with the patient's blood to prevent vasospasm. The Sim 2 (Merit Medical) was used to access the left common carotid artery with a guidewire for guidance. We used a Headway Duo (Microvention) and Aristotle 18 (Scientia) to access the left MMA under roadmap guidance, with successful embolization of the left MMA with Onyx 18 (Medtronic).

\section{Postoperative Course}

The patient returned to the neurology/neurosurgery intensive care unit after the procedure. No complications were reported during and after the procedure. The patient's neurological exam was significant for sixth cranial nerve palsy on the right. Her headache continued to improve over the next few days and completely resolved before discharge. The patient was started on a low-intensity heparin drip without bolus on day 3 postembolization because her platelet count continued to rise $\left(>100,000 / \mathrm{mm}^{3}\right)$. CT of the head demonstrated stable SDH. The patient was monitored in the neurological intensive care unit with 
hourly neurological checks for the first 4 days. The patient was switched from a heparin drip to apixaban 5 days post-procedure, and ATRA was restarted. The patient was then discharged and has since followed up with oncology and ophthalmology with no new complaints. She was later placed back on arsenic during her hematology/oncology follow-up appointment.

\section{Discussion}

\section{Literature Review Results}

Our search yielded a total of 7 articles representing 6 cases of cerebral sinus thrombosis associated with APL and/or ATRA therapy. Most of these case reports described coagulopathy secondary to APL, which further worsened upon initiating ATRA (4 cases). ${ }^{11,19-22}$ The patients were treated with early anticoagulation, except for 1 case in which CVT was associated with cerebellar hemorrhage and the patient was given fresh frozen plasma initially and then continued on anticoagulation. ${ }^{22}$ Most of the case reports recommended using heparin over warfarin, particularly during the induction and consolidation therapy because of concerns about higher risks of bleeding associated with thrombocytopenia and treatment-related coagulopathy. In one of the studies, researchers suspected that the thrombotic events were more common during the ATRA syndrome than during ATRA itself. ${ }^{11}$ Apart from these case reports, a retrospective study reviewed 34 patients with APL on presentation and during the induction period. There was no association found between hemostatic parameters in predicting either hemorrhage or thrombosis. After multivariate analysis, bleeding complications did not correlate with hemostatic parameters but did correlate with white cell count at presentation. ${ }^{23}$ The remaining reports described CVT secondary to AML, and the patients were treated with anticoagulation, except for 1 unique case report in which suction thrombectomy was performed. ${ }^{24-27}$

\section{Observations}

Several studies have described hematological malignancies as a risk factor for developing cerebral venous sinus thrombosis. ${ }^{28-30}$ Even though systemic anticoagulation is recommended as first-line therapy, venous sinus thrombectomy may be essential if CVT is refractory to medical therapy or if the patient's clinical course deteriorates secondary to cerebral edema or hemorrhage.

A promising outcome was achieved in the present case through MMA embolization despite the massive clot burden and additional challenges presented by the intracranial hemorrhages. A multidisciplinary discussion including intensive care, hematology/oncology, neurology, and neurosurgical services should occur regarding anticoagulation versus acute endovascular intervention in any patient with cerebral sinus venous thrombosis. MMA embolization has been demonstrated to be an alternative for chronic SDH management; ${ }^{31}$ however, limited experience has been documented in acute SDH. Nevertheless, MMA embolization was considered a safer approach in this patient with multiple comorbidities. This technique is usually well tolerated and carries a lower risk of complications, especially in patients with severe thrombocytopenia. Despite this excellent outcome, individual patient-based management protocols need to be shaped to balance the risks and the benefits of each case.

\section{Lessons}

To our knowledge, this is the first documented case treated with MMA embolization for an acute SDH in the setting of CVT in a patient with APL. Hemorrhagic and thrombotic complications are frequent in these patients. A multidisciplinary approach is essential to improve outcomes. Less invasive techniques such as endovascular interventions seem to be safe and effective. This case highlights the infinite scope of endovascular intervention as a potentially lifesaving measure and stimulates further investigation.

\section{References}

1. Tallman MS, Kwaan HC. Reassessing the hemostatic disorder associated with acute promyelocytic leukemia. Blood. 1992;79(3):543-553.

2. Falanga A, Barbui T. Coagulopathy of acute promyelocytic leukemia. Acta Haematol. 2001;106(1-2):43-51.

3. Huang ME, Ye YC, Chen SR, et al. Use of all-trans retinoic acid in the treatment of acute promyelocytic leukemia. Blood. 1988;72(2): 567-572.

4. Fenaux $\mathrm{P}$, Castaigne $\mathrm{S}$, Dombret $\mathrm{H}$, et al. All-transretinoic acid followed by intensive chemotherapy gives a high complete remission rate and may prolong remissions in newly diagnosed acute promyelocytic leukemia: a pilot study on 26 cases. Blood. 1992;80(9): 2176-2181.

5. Mandelli F, Diverio D, Avvisati G, et al. Molecular remission in PML/ RAR $\alpha$-positive acute promyelocytic leukemia by combined all-trans retinoic acid and idarubicin (AIDA) therapy. Blood. 1997;90(3):10141021.

6. Castaigne S, Chomienne C, Daniel MT, et al. All-trans retinoic acid as a differentiation therapy for acute promyelocytic leukemia. I. Clinical results. Blood. 1990;76(9):1704-1709.

7. Falanga A, Marchetti M, Barbui T. All-trans-retinoic acid and bleeding/thrombosis. Pathophysiol Haemost Thromb. 2003;33(suppl 1): 19-21.

8. Rashidi A, Silverberg ML, Conkling PR, Fisher SI. Thrombosis in acute promyelocytic leukemia. Thromb Res. 2013;131(4):281-289.

9. Tallman MS, Andersen JW, Schiffer CA. All-trans-retinoic acid in acute promyelocytic leukemia. N Engl J Med. 1997;337(15): 1021-1028

10. Escudier SM, Kantarjian HM, Estey EH. Thrombosis in patients with acute promyelocytic leukemia treated with and without all-trans retinoic acid. Leuk Lymphoma. 1996;20(5-6):435-439.

11. Fenaux $P$, Le Deley MC, Castaigne $S$, et al. Effect of all transretinoic acid in newly diagnosed acute promyelocytic leukemia. Results of a multicenter randomized trial. Blood. 1993;82(11):3241-3249.

12. Di Bona E, Avvisati G, Castaman G, et al. Early haemorrhagic morbidity and mortality during remission induction with or without alltrans retinoic acid in acute promyelocytic leukaemia. $\mathrm{Br} J$ Haematol. 2000;108(4):689-695.

13. Farge D, Debourdeau $P$, Beckers $M$, et al. International clinical practice guidelines for the treatment and prophylaxis of venous thromboembolism in patients with cancer. $J$ Thromb Haemost. 2013;11(1):56-70.

14. Lyman GH, Khorana AA, Kuderer NM, et al. Venous thromboembolism prophylaxis and treatment in patients with cancer: American Society of Clinical Oncology clinical practice guideline update. $J$ Clin Oncol. 2013;31(17):2189-2204.

15. Watson HG, Keeling DM, Laffan M, et al. Guideline on aspects of cancer-related venous thrombosis. Br J Haematol. 2015; 170(5):640-648.

16. Einhäupl KM, Villringer $A$, Meister $W$, et al. Heparin treatment in sinus venous thrombosis. Lancet. 1991;338(8767):597-600.

17. de Bruijn SF, Stam J. Randomized, placebo-controlled trial of anticoagulant treatment with low-molecular-weight heparin for cerebral sinus thrombosis. Stroke. 1999;30(3):484-488.

18. Coutinho JM, Ferro JM, Canhão P, et al. Unfractionated or lowmolecular weight heparin for the treatment of cerebral venous thrombosis. Stroke. 2010;41(11):2575-2580. 
19. Lee KR, Subrayan V, Win MM, et al. ATRA-induced cerebral sinus thrombosis. J Thromb Thrombolysis. 2014;38(1):87-89.

20. Ciccone M, Rigolin GM, Viglione GM, et al. Thrombosis of the cerebral veins and sinuses in acute promyelocytic leukemia after all-trans retinoic acid treatment: a case report. Blood Coagul Fibrinolysis. 2008;19(7): 721-723.

21. Song LX, Lu HY, Chang CK, et al. Cerebral venous and sinus thrombosis in a patient with acute promyelocytic leukemia during all-trans retinoic acid induction treatment. Blood Coagul Fibrinolysis. 2014;25(7):773-776.

22. Beslow LA, Abend NS, Smith SE. Cerebral sinus venous thrombosis complicated by cerebellar hemorrhage in a child with acute promyelocytic leukemia. J Child Neurol. 2009;24(1):110-114.

23. Dally N, Hoffman R, Haddad N, et al. Predictive factors of bleeding and thrombosis during induction therapy in acute promyelocytic leukemia-a single center experience in 34 patients. Thromb Res. 2005;116(2):109-114.

24. Haider AS, Sumdani H, McCaslin J, et al. Aggressive endovascular management of massive dural venous sinus thrombosis in the setting of acute myeloid leukemia. Cureus. 2019;11(1): e3891.

25. Tang ASO, Yeo ST, Law WC, Chew LP. Cerebral venous thrombosis as an initial manifestation of acute myeloid leukemia. Oxf Med Case Rep. 2019;2019(1):omy118.

26. Navaratne S, Blakeley CJ, Hashemi K. Superior sagittal sinus thrombosis, an unusual presentation of acute myeloid leukaemia: a case report. Emerg Med J. 2005;22(8):586-589.

27. Cabral de Andrade G, Lesczynsky A, Clímaco VM, et al. Cerebral venous sinuses thrombosis in both transverse sinus and torcula: multistep endovascular treatment and stenting. Interv Neuroradiol. 2017;23(1):84-89.
28. Paner A, Jay WM, Nand S, Michaelis LC. Cerebral vein and dural venous sinus thrombosis: risk factors, prognosis and treatment-a modern approach. Neuroophthalmology. 2009;33(5): 237-247.

29. Saadatnia M, Fatehi F, Basiri K, et al. Cerebral venous sinus thrombosis risk factors. Int J Stroke. 2009;4(2):111-123.

30. Thorell SE, Parry-Jones AR, Punter M, et al. Cerebral venous thrombosis-a primer for the haematologist. Blood Rev. 2015;29(1): 45-50.

31. Srivatsan A, Mohanty A, Nascimento FA, et al. Middle meningeal artery embolization for chronic subdural hematoma: meta-analysis and systematic review. World Neurosurg. 2019;122:613-619.

\section{Disclosures}

The authors report no conflict of interest concerning the materials or methods used in this study or the findings specified in this paper.

\section{Author Contributions}

Conception and design: all authors. Acquisition of data: Maini, Afzal, Giurgiutiu, Shah. Analysis and interpretation of data: Maini, Giurgiutiu. Drafting the article: Maini, Giurgiutiu, Switzer. Critically revising the article: Maini, Afzal, Giurgiutiu, Shah, Switzer, Vale, Garcia. Reviewed submitted version of manuscript: Maini, Giurgiutiu, Rahimi, Vale, Garcia. Approved the final version of the manuscript on behalf of all authors: Maini. Administrative/technical/material support: Rahimi. Study supervision: Giurgiutiu, Rahimi.

\section{Correspondence}

Kushagra Maini: Augusta University, Augusta, GA. kmaini@augusta.edu. 